\title{
Introduction chapter
}

\section{Cilia Witteman • Wiebe van der Hoek}

Received: 12 September 2012 / Accepted: 20 September 2012 / Published online: 27 September 2012 (C) Springer Science+Business Media Dordrecht 2012

\section{Introduction}

In the Spring of 2011 we sent out a call for papers for a special issue of Knowledge, Rationality and Action, a special section of Synthese, on Psychological Models of (Ir)rationality and Decision Making. Both empirical and conceptual papers were welcome.

We were happy to receive over twenty abstracts of proposed papers. In the Summer of 2012 we ended up with a very nice collection of fourteen peer-reviewed papers. The majority of the papers in this special issue (nine) is conceptual, five papers describe empirical studies.

The conceptual papers address topics ranging from utilities and preferences to predictions, from causal reasoning to group decisions, with approaches ranging from more philosophical to computational modelling.

Weiss and Weiss, in Irrational-at the moment, argue that with everyday decisions, considerations can change quickly, and thus that what seems irrational, because it is inconsistent with a previously established policy, need not be, since it need not violate a personal policy. The contribution presents a descriptive multi-attribute model, which includes momentary salience as a parameter and in which little everyday decisions are nested under big decisions, and therefore need not be thought about extensively again.

Alfano, in Wilde heuristics and Rum Tum Tuggers, addresses a similar topic: the stability of preferences. This papers reminds us that in rational decision theory and game theory preferences are assumed to be stable and transitive, but that actual behaviour

\footnotetext{
C. Witteman $(\varangle)$

Behavioural Science Institute, Radboud University, Nijmegen, The Netherlands

e-mail: C.Witteman@socsci.ru.nl

W. van der Hoek

The University of Liverpool, Liverpool, UK
} 
shows that they need not be. That poses a problem for these theories, which the author proposes may be resolved by a model in which preferences are locally unstable and indeterminate but approximate stability and determinacy when the differences between options become large.

Hagmayer and Osman, in From colliding billiard balls to colluding desparate housewives, introduce causal Bayes nets as rational models to describe people's everyday causal reasoning. The paper's starting observation is that many decision theories do not talk about causal reasoning, while this is a basic type of inference. The reasoning here is illustrated by referring to the television series Desperate Housewives, where we understand the protagonists by inferring their motives and intentions through causal reasoning.

Harris and Osman discuss the phenomenon of 'illusion of control': perceiving a causal relationship between one's own action and an outcome where none exists. The authors argue that when there is a choice in a task, people assume agency over outcomes. Using the London 2011 riots as an example, the authors show that assuming an uncontrollable situation to be controllable is quite rational, and that perceived control can affect real world outcomes (i.e. the end of rioting).

Elqayam presents a paper on grounded rationality, a meta-theoretical framework based on both descriptivism and epistemic relativism. Grounded rationality accepts universal human biological and cognitive limitations, to which it adds a recognition of cognitive variability within and between people.

Slavkovik and Boella propose a model for group decision making. This model is an extension of the well-researched Recognition-Primed Decision (RPD) approach, where a decision follows upon recognition of the situation as one seen previously. This extension they call Recognition-Primed group Decisions (RPgD). The authors give a formal description of how, in their model, information from different agents can be aggregated.

Hotaling and Busemeyer discuss an extension of Decision Field Theory (DFT), which assumes that subjective evaluations of alternatives are accumulated until a threshold is reached, to account for dynamic decision making, DFT-D. They show that this model is capable of capturing individual strategies in dynamic decisions that are traditionally taken to lead to irrational choice.

Rosenfeld, Zukerman, Azaria and Kraus propose machine learning algorithms as complements to psychological models based on bounded rationality, to predict people's decisions. They demonstrate that the resulting hybrid methods predict decisions quite well even in complex domains such as negotiation.

Dickert, Västfjäll, Kleber and Slovic talk about the valuation of human lives. One of their interesting and at first sight counter-intuitive findings is that as the number of victims that we are asked to help increases, our willingness to do so decreases. The authors propose a descriptive model to explain this phenomenon, which takes into account different motivations and conditions under which people deviate from the normative, egalitarian valuation of human lives.

The next set of five papers has a more empirical focus. The first of these papers, by Baron, Gurcay, Moore and Starcke links to the conceptual paper by Dickert et al., also discussing moral judgments. Baron et al. address individual differences in reactions to moral dilemmas. They propose a model that combines a subject's ability to give 
utilitarian (consequence-based) answers and the difficulty of giving such answers to moral dilemmas. Applying a Rasch (mathematical) model to different data sets indeed shows interesting response time differences for different subjects and different dilemmas.

Aarts, Witteman, Souren and Egger study individual differences in decision making by professionals, i.e., clinical psychologists. They assess the clinicians' decision making styles and preferences, and show that these differed among clinicians and also that they are more predictive of decision accuracy than the clinicians' years of experience.

Ayal, Zakay and Hochman, in Deliberative adjustments of intuitive anchors, also examine individual differences. They looked at differences between students, in intuitive and rational thinking styles and their relation to diversification behaviour in everyday situations. They report that students with a deliberative thinking style are more calibrated to normative, rational behaviour.

Jekel, Glöckner, Fiedler and Bröder, in The rationality of different kinds of intuitive decision processes, focus on probabilistic decision tasks. They compare the performance of different simulated strategies underlying intuition, to the rational solution calculated with Bayes' theorem. They conclude that parallel constraint satisfaction, a network model of continuous automatic information re-evaluation until a coherent representation is reached, mimics the rational solution best.

Finally, Dhami and Mandel, in Forecasted risk taking in youth, have asked young people about the perceived importance of the benefits and the drawbacks of risky behaviours and the expected probabilities of the outcomes of different behaviours separately. They found that the only significant predictor of engaging in risky behaviour was the perceived importance of the benefits of that behaviour: a simple, non-compensatory model. It is useful for policy makers to know that young people hardly take expected drawbacks into account.

We trust that with this collection of papers we have given both philosophical and psychological researchers of (ir)rationality and decision making some interesting food for thought. 